\title{
Perspectives on the draft ICH-M10 guidance: an interview with Mark Arnold
}

\author{
Mark E Arnold*,1 \\ ${ }^{1}$ Covance, Inc., Princeton, NJ 08540, USA \\ *Author for correspondence: mark.arnold2@covance.com
}

Biography: Mark E Arnold, PhD, is Director of Science for Covance Laboratories. In that role, he develops the bioanalytical strategy for immune-, cell-based, quantitative polymerase chain reaction (qPCR) and LCMS/MS assays to quantify drugs and metabolites, antidrug antibodies and biomarkers in animal and clinical samples for pharmacokinetic and pharmacodynamic assessments. Mark was previously Executive Director of Bioanalytical Sciences at Bristol-Myers Squibb. He received a BS (biology) from Indiana University of Pennsylvania and PhD (pharmacology) from the University of Pittsburgh. For more than 30 years, Mark has been involved in the evolving field of bioanalysis, including the science and the review and interpretation of regulations and guidance. He co-chaired the AAPS Crystal City V and VI Workshops on the US 'FDA Draft Revised Guidance on Bioanalytical Method Validation' and 'Biomarkers'. He is actively involved in the Land O'Lakes Bioanalytical Conference and American Association of Pharmaceutical Scientists (AAPS, named Fellow in 2014). Mark has over 100 peer-reviewed publications, and numerous invited podium presentations.

This interview was conducted by Sankeetha Nadarajah, Managing Commissioning Editor of Bioanalysis.

Accepted for publication: 15 July 2019; Published online: 6 September 2019

Keywords: guidance $\bullet \mathrm{ICH}-\mathrm{M} 10 \bullet$ pivotal $\bullet$ regulation $\bullet$ scope

\section{Can you provide a brief overview of the scope of the draft ICH-M10 guidance?}

Interpreting the scope as written in the draft M10 guidance has been one of the most challenging aspects for the bioanalytical community in reviewing the document. It does simplify the primary matrix and primary analyte assays as those requiring full validation and permits some metabolites, tissues and additional matrices to use methods with fit-for-purpose (partial) validations. That simplification, however, is countered by the use of the term 'pivotal' for studies that have higher requirements. The definition of 'pivotal' has evolved over the years and may have its broadest and most impactful implementation in ICH-M10. The 2011 EMA bioanalytical method validation guideline used the term in relation to bioequivalence studies; which all agree require the most extensive full validation. The US FDA guidance (2018) expanded 'pivotal' to those studies providing data required in support of regulatory decisions (approval, safety or labeling); which from industry-agency discussions seemed to be a subset of all clinical studies and some of the Good Laboratory Practice (GLP) toxicokinetic (TK) studies.

ICH-M10 is similar to the FDA guidance, in that GLP TK studies used in regulatory decision-making are included, but expands included clinical trials to 'all phases of clinical trials in regulatory submissions'. This clause for clinical trials seems to require full validation for the primary analyte/matrix for all clinical studies since all clinical trials are typically included within a regulatory submission. Industry organizations (e.g., American Association of Pharmaceutical Scientists, China Bioanalysis Forum, European Bioanalysis Forum, Japan Bioanalysis Forum) are seeking clarity on which clinical studies require full validation and laboratory execution similar to bioequivalence studies through their comments to their local health authority that are passed on to the ICH.

\section{Why was there a need for the ICH-M10 guidance, when there is already BMV guidance available?}

Differences in regional documents, both when implemented as guidance or as local regulatory law, have made it challenging to perform work that can be accepted globally. Activities to define a sound and globally accepted scientific basis for regulated bioanalysis have been ongoing for nearly a decade through a variety of organizations like 
those listed above along with the Global Bioanalysis Consortium and Applied Pharmaceutical Analysis. Through initiatives led by these organizations, as well as through bioanalytical meetings - including the Land O'Lakes Bioanalytical and Workshop on Recent Issues in Bioanalysis conferences - regulators and industry counterparts have come together to openly discuss the science and regulations in an effort to define expectations and best practices. Through these efforts, there are much greater levels of communication and transparency for regulated bioanalysis and, while adopting the core practices, each country and region continues to have expectations that are unique.

Combined, these differences have significantly expanded the work needed to develop single bioanalytical packages that can be used globally as part of new drug and generic drug applications; thereby, contributing to increased costs in bringing a new drug to market. Achieving an ICH guidance in M10 that will be accepted in so many countries and regions will establish a common set of expectations, reduce some of the unique differences and allow for the more efficient generation of data for new drug approvals for patients.

\section{Can you explain the differences between a full, partial and cross validation, and how you decide which one is appropriate to specific studies?}

The key thing to remember with what I am about to discuss is that this is based on the current draft guidance and is subject to change. As mentioned before, full validations are required for assays of the primary analyte(s) in the primary matrix for TK studies and pivotal clinical studies (including bioavailability [BA]/bioequivalence [BE]) that provide data that support regulatory decisions. That places some metabolites and additional matrices in the category of requiring only a partial validation. The data from these assays would help characterize the presence of the drug but would not be essential to a regulatory decision. One interesting aspect is that partial validations may also be used if a fully validated assay is used for another matrix or the same matrix in another species. Questions have been raised as to whether this permits, for example, a rat plasma assay used to support an investigational new drug (IND) toxicity study can be partially validated for an IND-supporting toxicity study in dogs. Another use for partial validations is for a demonstrating that minor changes to a fully validated method have not introduced impactful changes to the method.

Cross validations are another area of the draft guidance in which there are several options. In this case, however, the requirements are to ensure that the data generated within a study or between studies may reliably be compared. The three cases defined within M10 are: when two methods are used in a single study; when different methods are used in different studies; or when samples from a study are analyzed in two labs using the same method. Industry scientists are in agreement that the first and third situations make sense but several reasons have been discussed for not endorsing the second. One key challenge is that intersubject variability is typically higher than assay variability, rendering comparisons of the methods less valuable. Additionally, the current drug development paradigm often involves transfer of a drug between companies (through direct purchase of the drug or company mergers) or running the assay in multiple labs, both resulting in multiple labs and assays being used over the course of the development program. It is difficult, therefore, to interpret the relationship between the earliest and final studies across the series of cross-validations that would be required in such a scenario.

\section{Are there any disadvantages of such a collective consensus guidance?}

Any new guidance, whether it is country-specific, regional or multiregional; is put together by committee and is based upon the experiences of the participants in defining what is critical and of value. As expected, the core principles going into the ICH Expert Working Group (EWG) deliberations were fairly consistent and remained consistent in the draft. Participants in the EWG were given a challenging task and have spent extensive time and effort in developing the draft guidance. It seems likely that only a portion of the issues discussed were deemed worthy of inclusion in the final document by the entire EWG. That said, some of the individual regional points of difference seem to have been added together and make the harmonized requirements of the draft more extensive than most country's/region's current regulations. As there has been clarification and simplification of some aspects of the document, the final version of the guidance will need to be reviewed to fully understand impact. With what is expected to be extensive comment from various pharmaceutical companies, contract research organizations (CROs) and scientific organizations, it is premature to guess on the final impact. 


\section{How easily can global laboratories adapt to implement the ICH-M10 guidance?}

If the draft guidance is not substantially changed when it comes out as final, it should not take significant effort to change practices to comply as many labs had already included many of the country- and region-specific requirements around validation and sample analysis, especially for BE studies. There is one exception: the comprehensive reporting requirements within the draft (for what appears to be most clinical trials) will necessitate developing new practices and software to include additional required content in study reports that had previously been required to be maintained at the laboratory site. With global pharmaceutical companies and their CRO partners having common needs, it will present an opportunity for software vendors to respond quickly with new versions that can deliver the newly required report content.

There were discussions at the recent AAPS ICH-M10 Workshop around the formation of an industry consortium to work with health authorities to establish a bioanalytical data standard, similar to Standard Exchange for Nonclinical Data (SEND), that could provide data in an electronic format for regulatory review and not require extensive additional tables of information to each nonclinical or clinical report. Such a standard would simplify how software vendors transfer their content to a common format, making the submission process more efficient and also saving pharmaceutical and CRO firms from having to redundantly recreate, at high cost, the needed software to format data to the standard.

\section{Will the ICH-M10 guidance meet the expectation from industry on a harmonized consensus guidance?}

In short, yes. By setting a common standard as a global set of regulatory expectations, M10 offers a stable set of practices enabling more efficiency, and aligned tools and processes to be developed. Given the historically slow pace of ICH guidance revisions, it will be difficult to implement rapid changes and, thus, investments will have longer periods over which to retain value. For each laboratory, this will also translate into reducing the need to change SOPs or adapt report content to address regional requirements. The potential may then exist for industry consortia or scientific organizations to collaborate in developing commonly accepted report templates and data file formats. These actions will all drive efficiencies for the laboratories. The one unknown, related to industry expectations for the final harmonized guidance, is how extensive the set of requirements will be as compared with today's existing regulations, especially around the breadth of the term 'pivotal' as applied to clinical studies.

\section{How will the ICH-M10 guidance change the bioanalytical regulatory landscape?}

A cascade of events becomes possible:

- Clarity and consistency on globally acceptable practices is feasible;

- Mutually acceptable data standards may actually be achieved;

- Organizations like Pharmaceutical Inspection Co-operation Scheme (PIC/S); which has been working to provide training and harmonization in Good Manfacturing Practice (GMP) and Good Clinical Practice (GCP) inspection practices, can further expand their training and collaboration into GLP- and GCP-specific bioanalytical method validation bioanalytical laboratory inspections;

- Consistency in inspector expectations will lessen laboratory concerns during an inspection and will increase the acceptability of data globally from labs that have been inspected to a global standard, reducing the risk for pharmaceutical companies and CROs, alike.

\section{What have you learned so far from the discussions with regulators and industry peers that is} reshaping your understanding of the guidance and its future success?

Three things come to mind. First, the expanded reporting requirements are designed to reduce the need for regulatory reviewers to request content from the sponsor, which can delay the final review and approval of a filing. It may also reduce the need to send auditors out into the field to look for or audit data in a lab. These two aspects can accelerate drug filing reviews and approvals. Second, while regulators feel that conference attendees from both pharmaceutical companies and CROs are well-versed in and compliant with existing regulations, they also see that there are some people and organizations who do not fully comprehend their expectations. These gaps in understanding result in practices and data that may not be reliable enough for decision-making. Regulators respond by expanding guidance requirements to ensure the sufficiency and reliability of data. Clear and unambiguous language that both regulators and the scientific community understand in the same way will be needed in ICH- 
M10 to achieve their goal for broad understanding and high quality, reliable bioanalytical packages within filings. Third - and very welcome - is that the EWG will recommend ICH develop training to globally communicate the expectations specified within ICH-M10 to aid in its uptake by laboratories. This will help both sponsors and CROs achieve a common understanding of the guidance expectations. For CROs, this can also improve efficiencies as there will be fewer differences in expectations from their various sponsors.

\section{Disclaimer}

The opinions expressed in this interview are those of the interviewee and do not necessarily reflect the views of Covance Inc.

\section{Financial \& competing interests disclosure}

The interviewee is employed by a company that will be required to comply with the final ICH-M10 Guidance. The interviewee has no other relevant affiliations or financial involvement with any organization or entity with a financial interest in or financial conflict with the subject matter or materials discussed in the manuscript apart from those disclosed.

No writing assistance was utilized in the production of this manuscript. 